Meta

Journal des traducteurs

Translators' Journal

\title{
La synesthésie déconstruite ou l'intermédiaire traductionnel sourd au " bruit des couleurs " et insensible au " goût de la musique "
}

\section{Claudine Lécrivain et Elena Romero Alfaro}

Volume 46, numéro 3, septembre 2001

URI : https://id.erudit.org/iderudit/003439ar

DOI : https://doi.org/10.7202/003439ar

Aller au sommaire du numéro

Éditeur(s)

Les Presses de l'Université de Montréal

ISSN

0026-0452 (imprimé)

1492-1421 (numérique)

Découvrir la revue

Citer cet article

Lécrivain, C. \& Romero Alfaro, E. (2001). La synesthésie déconstruite ou l'intermédiaire traductionnel sourd au " bruit des couleurs " et insensible au " goût de la musique ». Meta, 46(3), 524-534. https://doi.org/10.7202/003439ar
Résumé de l'article

Le processus de lecture-écriture présent dans l'activité traductionnelle met à jour une sorte de désaccord sur la pertinence des propositions exprimées au moyen de la synesthésie, en tant que représentation d'une osmose sensorielle qui rend compte d'un ordre du monde possible dans les textes de fiction littéraire. Le comportement des intermédiaires traductionnels révèle, pour des raisons que cet article essaie d'élucider, soit un rejet de la synesthésie et donc l'inscription d'une expérience sensorielle habituelle, d'une cosmovision traditionnelle, soit une réorganisation du co-texte immédiat en sorte que le lecteur puisse inférer favorablement l'énoncé synesthésique et réduire son instabilité interprétative. 


\title{
La synesthésie déconstruite ou l'intermédiaire traductionnel sourd au «bruit des couleurs » et insensible au «goût de la musique»
}

\author{
CLAUDINE LÉCRIVAIN
}

ELENA ROMERO ALFARO

Université de Cádiz, Cádiz, Espagne

\begin{abstract}
RÉSUMÉ
Le processus de lecture-écriture présent dans l'activité traductionnelle met à jour une sorte de désaccord sur la pertinence des propositions exprimées au moyen de la synesthésie, en tant que représentation d'une osmose sensorielle qui rend compte d'un ordre du monde possible dans les textes de fiction littéraire. Le comportement des intermédiaires traductionnels révèle, pour des raisons que cet article essaie d'élucider, soit un rejet de la synesthésie et donc l'inscription d'une expérience sensorielle habituelle, d'une cosmovision traditionnelle, soit une réorganisation du co-texte immédiat en sorte que le lecteur puisse inférer favorablement l'énoncé synesthésique et réduire son instabilité interprétative.
\end{abstract}

\begin{abstract}
The reading-writing process in translational activity produces a certain element of disagreement on the pertinence of proposals expressed by means of synaesthesia-a representation of a sensorial osmosis which is conscious of a possible world order within the texts of literary fiction. The conduct of translational intermediairies reveals, for reasons this article intends to elucidate, either a rejection of synaesthesia and thus the acceptance of an habitual sensorial experience, of a traditionnal cosmovision, or a reorganisation of the immediate co-text in such a way as to enable the reader to favourably infer the synaesthesic enunciation and reduce its interpretative instability.
\end{abstract}

\section{MOTS-CLÉS/KEYWORDS}

création littéraire, figures de style, osmose sensorielle, processus de lecture-écriture, synesthésie

La création littéraire se fonde sur un rapport plus ou moins étroit avec le réel, constamment redéchiffré dans chaque œuvre, perçu et (re)structuré, avec une intensité différente à chaque fois. Dans la fiction, la représentation de personnages, objets ou événements propose divers degrés d'approximation et d'éloignement à la perception ordinaire d'un «réel objectif», synthèse d'une appréhension sensorielle, culturelle et linguistique. Je m'intéresserai ici à l'interprétation-lecture qui se manifeste dans l'acte traductionnel des énoncés de fiction où s'inscrit une assimilation dans la perception sensorielle. Je fais référence ici à la synesthésie, figure qui entremêle deux ou plusieurs sensations ${ }^{1}$ et qui entre le plus souvent dans une notation descriptive concernant essentiellement l'aspectualisation. Relevant «davantage de l'exercice affectif du langage que de mécanismes linguistiques spécifiques» (Bonhomme, 1998: 68), elle est le plus souvent présente sous la forme de caractérisation adjective ${ }^{2}$ et généralement restreinte à de brefs syntagmes. 
La synesthésie, comme d'autres figures, et notamment la métaphore ${ }^{3}$, ne présente pas «un écart par rapport à la norme ou une transgression d'une règle ou d'une maxime de communication» (Sperber et Wilson 1986: 24). Les analyses pragmatiques portant sur ce type d'énoncés soulignent bien qu'ils ne proposent pas un «sens figuré» qui viendrait se substituer à une signification littérale (Sperber et Wilson, 1986; Vicente Cruz, 1993) et que leur interprétation se fait par un processus identique à celui qui est à l'œuvre dans l'interprétation des énoncés ordinaires (Moeschler et Reboul 1994 : 416-422). Mais dans la mesure où il s'agit d'une exploitation créative de la communication verbale, le faisceau d'implications logiques et contextuelles qui devront être réalisées par le lecteur afin de compléter les lacunes ou les «relâchements» informationnels de l'énoncé sera plus intense et plus ample. La compréhension de ces énoncés ne passe donc pas, comme l'a indiqué la rhétorique traditionnelle, par une simple paraphrase qui permettrait de les saisir bien qu'imparfaitement et bien que partiellement, mais elle exploite des implications analytiques et contextuelles. Par conséquent, plus fort sera le degré de créativité de ces énoncés, plus leur interprétation sera instable, car les conclusions auxquelles arrivera le lecteur seront en relation directe avec ses connaissances et son imagination, qui ne sauraient être autres que singulières. L'interprétation de ces énoncés littéraires dépend donc des expériences cognitives de l'interlocuteur et du pacte portant sur l'une des conventions propres à la fiction, à savoir que les objets «n'ont pas à obéir aux lois physiques qui gèrent le comportement des objets dans le monde réel» (Moeschler et Reboul 1994: 439).

Maints romanciers et poètes ont ainsi utilisé cette combinaison de différentes modalités sensorielles dans la représentation de la perception faisant état d'une osmose sensorielle, impossible à segmenter. Je citerai ici l'exemple bien connu de Proust:

[...] nous entendions au bout du jardin, non pas le grelot profus et criard qui arrosait, qui étourdissait au passage de son bruit ferrugineux, intarissable et glacé [...] mais le double tintement timide, ovale et doré de la clochette pour les étrangers [...]. (Du côté de chez Swann: 18)

Si locuteur et interlocuteur partagent les mêmes conventions sur le discours de fiction, il est clair que ce fragment ne requiert pas une analyse qui permettrait de le paraphraser de façon satisfaisante, d'en extraire un effet principal réduisant l'incompatibilité sémantique du syntagme tintement ovale, proposant le rétablissement d'un rapport logique entre les termes tintement et ovale, ou d'un rapport linguistique qui passerait par une analyse componentielle conduisant à un contenu cognitif stable 4 . Face à la notation synesthésique, le processus interprétatif, quelle que soit la conclusion à laquelle il a abouti, admet finalement la juxtaposition de ces éléments qui rendent compte d'un ordre du monde possible sans qu'il y ait constatation de non-vérité de la proposition exprimée sur la base de «l'interprétation des énoncés immédiatement précédents, les connaissances encyclopédiques dont nous disposons sur le monde, l'environnement physique» (Moeschler et Reboul 1994: 418-4195).

On devrait donc s'attendre à ce que, face à cette image arbitraire qui propose une sorte de plasticité auditive, le traducteur ait une réponse traductionnelle presque automatique qui consisterait en une traduction à dominante littérale des différentes expressions qui résultent de la condensation et de la simultanéité des perceptions, en vue de reproduire dans le texte-cible les possibilités d'un apparentement, d'établir un 
système de relations sensorielles à l'intérieur du réel fictif. Puisque le traducteur n'est pas aux prises avec des écueils proprement linguistiques (comme cela pourrait être le cas lors de la traduction d'une syllepse proposant un cumul de significations sur un même signifiant: sens propre et sens figuré), sa réécriture devrait donc proposer à son tour la même perception particulière de la réalité que celle du texte-source. Telle est la démarche de Pedro Salinas lorsqu'il maintient le transfert sensoriel de tintineo oval:

[...] oíamos al extremo del jardín, no el cascabel chillón y profuso que regaba y aturdía a su paso con un ruido ferruginoso, helado e inagotable [...] sino el doble tintineo, tímido, oval y dorado de la campanilla, que anunciaba a los de fuera $[. .$.$] . (Por el camino$ de Swann: 24-25)

Or le processus de lecture-écriture présent dans l'activité traductionnelle met à jour une sorte de désaccord/discordance sur la pertinence des propositions exprimées au moyen de la synesthésie, pour des raisons et par des moyens que j'essaierai d'élucider. Les recherches menées sur un corpus restreint (exclusivement des textes en prose) qui puisse s'adapter au cadre de cet article ont démontré que bien souvent un redressement apparaît, redressement que l'on peut sans doute imputer aux traducteurs, mais aussi aux réviseurs, correcteurs, éditeurs, donc à un «intermédiaire traductionnel» qui considère ou bien que les propos de l'auteur ne sont pas vériconditionnels ou bien qu'ils seront trop difficilement accessibles au lecteur du texte traduit, ou encore que, même s'ils sont accessibles, ils vont constituer un ralentissement de la lecture et de la compréhension. Il s'avère alors que cet intermédiaire impose dans certains énoncés non pas sa propre expérience perceptuelle, mais une expérience sensorielle habituelle, une cosmovision traditionnelle dans la présentation de «la façon dont les choses du monde s'apparentent ou se distancent, leur manière de se construire, de se combiner, de se séparer, de s'attirer de se repousser les unes les autres» (Arnheim 1976: 40) comme si l'univers esthétique fondé sur les relations sensorielles présent dans le texte-source n'était pas suffisamment ressemblant. Lintermédiaire traductionnel introduit aussi parfois des modifications qui vont organiser le cotexte immédiat en sorte que le lecteur puisse inférer favorablement l'énoncé, et qui vont servir d'appui à l'interprétation des segments synesthésiques, dans un souci de réduction de leur instabilité interprétative, et donc dans un souci de garantir au lecteur-cible «l'accès au texte», comme s'il était unique et s'épuisait dans le rapport à un univers objectif. La traduction s'avère ainsi une activité privilégiée où se joue le rapport à la lecture et à la littérature, rapport qui se voit ainsi indirectement questionné. Les constatations qui dérivent de l'analyse des textes traduits éclaireront donc parallèlement et l'activité traductionnelle ${ }^{6}$ et l'activité lectrice.

Les extraits suivants proposent donc, en guise d'échantillon, des exemples pour lesquels, à chaque fois qu'une traduction littérale ou à dominante littérale était possible, l'intermédiaire traductionnel a choisi de rejeter la stratégie littérale et d'effacer à différents degrés l'inscription de la fusion de différentes expériences sensorielles dans une image poétique.

Dans les exemples 1-6 les auteurs proposent des synesthésies « arbitraires $^{7} »$ qui rendent sensibles, qui «donnent à voir » des impressions, dans une transmutation de sensations : 
(1) Gracias a ella, los pisos de tierra golpeada, los muros de barro sin encalar, los rústicos muebles de madera construidos por ellos mismos estaban siempre limpios, y los viejos arcones donde se guardaba la ropa exhalaban un tibio olor de albahaca. (Cien años de soledad: 13)

Par ses soins, le sol de terre battue, les murs de boue séchée qu'on n'avait pas blanchis à la chaux, les meubles rustiqurs qu'ils avaient fabriqués eux-mêmes demeuraient toujours propres, et les vieux coffres où l'on gardait le linge dégageaient une bonne odeur de basilic. (Cent ans de solitude: 17)

(2) Aquel olor mordiente quedaría para siempre en su memoria, vinculado al recuerdo de Melquiades (Cien años de soledad, 11)

Cette odeur âcre devait rester à jamais dans sa mémoire, inséparable du souvenir de Melquiades (p. 14)

Dans les deux exemples précédents la synesthésie olfactif/tactile (que l'on aurait pu formuler par «odeur douceâtre» et «odeur cuisante $\left.{ }^{8} »\right)$ est neutralisée par l'intermédiaire traductionnel qui privilégie une adjectivation compatible avec l'odeur. L'effacement de la synesthésie révèle ici un rejet de l'opacité immédiate du segment concerné et une imposition du bon usage littéraire de la description lié à une représentation, courante et vraisemblable, des structures du réel.

(3) Et partout, sur sa tête, l'égouttement froid, les petites notes salées des cloches de la paroisse, projetées comme d'un goupillon pour quelque absoute. (Bruges-la-morte: 26) Y por todas partes sentía sobre su cabeza la llovizna fría, el desgrane de las leves notas de las campanas parroquiales que caían como desde un hisopo que absuelve. (Brujas, la muerta: 32)

Là encore, la synesthésie auditif/gustatif est neutralisée par une adjectivation compatible et l'inscription d'une perception concrète de transferts sensoriels effacée. Ces effacements laissent déjà entrevoir des automatismes de la perception et de la représentation chez ce lecteur professionnel qu'est l'intermédiaire traductionnel. Les propriétés évocatoires disparaissent au profit de propriétés purement descriptives, comme si soudainement les artifices verbaux ne pouvaient exister qu'au service d'un ordre naturel, d'une «illusion du vrai». Cette description qui semblerait alors ne pouvoir valoir fondamentalement que comme possibilité de référence vis-à-vis du monde extérieur ${ }^{9}$ conduit à percevoir les syntagmes synesthésiques comme des défaillances de la perception; alors que la fiction, plus ou moins articulée sur la réalité, est une mise à l'épreuve des frontières de l'imaginaire et ne demande pas à être corrigée mais seulement interprétée. Or l'intermédiaire traductionnel semble plus sensible, d'une part, à une cohérence du domaine référentiel, mettant en pratique une correction des "déviances» référentielles, et d'autre part à une modalité traditionnelle de lecture basée sur la dichotomie sens littéral/sens figuré, et lorsqu'il se trouve face à des énoncés qu'il considère irréductibles, il procède à inscrire une interprétation en relation avec la description conventionnelle ou avec un soi-disant «vouloir-dire» de l'auteur qui sera signifié explicitement afin de garantir la stabilité de l'interprétation:

(4) Et elle rentrait par les rues mortes, regrettant la verte banlieue de l'aube, la messe, les cantiques blancs, toutes les choses sur lesquelles la nuit tombait. (Bruges-la-morte: 66) Y recorría las calles muertas, recordando con tristeza el risueño paisaje del alba, la misa, los cánticos celestiales, todas las cosas sobre las que la noche caía. (Brujas, la muerta: 96-97) 
La démarche créatrice présente dans le texte original est une fois encore annulée par le redressement de la compatibilité sémantique substantif-adjectif qui vient cautionner la véracité des descriptions. L’intermédiaire traductionnel refuse la simultanéité des sens, l'unité du monde perçu (à la fois fragmentation et synthèse) et annule le transfert de signification en proposant un qualificatif courant, proche du cliché. D'ailleurs dans le même énoncé a lieu la transformation d'un autre adjectif de couleur ( «la verte banlieue de l'aube/ el risueño paisaje del alba»), comme si ces deux références à la couleur n'étaient pas suffisamment stables en vue de l'effet proposé et demandaient une précision qui facilite le processus de lecture. Phénomène identique dans la traduction de l'extrait suivant où le choix d'un terme polysémique (matiz) faisant référence à l'impression auditive et visuelle, débouche sur une interprétation privilégiant l'impression auditive d'autant plus que la métaphore «voix orfévrée» est également neutralisée:

(5) La voix aussi! La voix de l'autre, toute semblable et réentendue, une voix de la même couleur, une voix orfévrée de même. (Bruges-la-morte: 39)

¡ La voz también! La voz de la otra, igual, sonando de nuevo; una voz del mismo matiz y timbrada lo mismo. (Brujas, la muerta: 51)

Les synesthésies des exemples 6-8 sont moins arbitraires que celles des exemples précédents dans la mesure où les implications cotextuelles donnent lieu à une interprétation nettement plus stable dans le cadre du pacte discursif entre locuteur et interlocuteur pour faire exister une scène imaginaire.

(6) Dans la chambre voisine, j'entendais ma tante qui causait toute seule à mi-voix [...] Au bout d'un moment, j'entrais l'embrasser [...] Elle tendait à mes lèvres son triste front pâle et fade sur lequel, à cette heure matinale, elle n'avait pas encore arrangé ses faux cheveux [...]. (Du côté de chez Swann: 61-63)

En el cuarto de la lado oía a mi tía hablar ella sola a media voz [...] Al cabo de un momento entraba a darle un beso [...] Ofrecía a mis labios su frente pálida y fría, que en aquellas horas tempranas aún no tenía puestos los postizos [...]. (Por el carmino de Swann: 68-70)

Cet extrait propose une combinaison de synesthésie et d'hypallage ${ }^{10}$ : bien que le rapport linguistique soit absent dans le syntagme son triste front pâle et fade, il existe néanmoins un rapport logique contextuel qui explique le déplacement qualificatif. En effet la synesthésie, fondée sur la correspondance au niveau de la perception entre une représentation visuelle ou tactile (front) et une perception gustative (fade) n'est pas étrangère à l'isotopie du contexte, car elle s'intègre dans le champ associatif de lèvres. D'autre part, l'attribution au front d'une sensation gustative n'est pas vraiment impertinente, ni illogique. Il s'agit plutôt d'une attribution peu fréquente. La traduction de Pedro Salinas propose cette fois un redressement du «degré de convenance ", un retour à une perception traditionnelle, inscrivant la sensation tactile (fría), l'attribution courante de la qualité de froid ou de chaud au front, au détriment de la sensation gustative du texte-source (fade). L'intermédiaire traductionnel rejette le terme discordant par rapport à certaines conventions descriptives, recherchant parallèlement une "cohérence» et un stéréotype. Puisque front fade n'est ni plus ni moins exact que frente fría, et que ni l'une ni l'autre des deux expressions ne s'éloigne d'une description coïncidente avec le monde réel, la reformulation ne répond plus ici à un souci de vraisemblance, mais témoigne de la force de ce cliché descriptif qui semble resurgir spontanément. 
Ce type de médiation réductrice est également opéré par l'intermédiaire traductionnel de l'extrait 7 pour qui, même si l'expérience permet d'affirmer que certains objets dégagent une odeur lorsqu'ils sont mouillés ou bien chauds, il ne saurait être question de caractériser cette odeur par une correpondance connexe, une simultanéité sensorielle olfactive et gustative (olor insipido/insulso) :

(7) On ne voyait rien, mais on sentait la froideur hostile de l'eau et l'odeur fade des pierres mouillées (Les misérables, vol. III : 359)

No se veía nada; pero se sentía la frialdad hostil del agua y el olor especial de las piedras mojadas (Vol II : 1160)

(8) Et, à mesure qu'on avance dans le mois d'août et que le soleil grandit, le blanc des maisons se fait plus aveuglant et les peaux prennent une chaleur plus sombre. (Noce: 37)

Y, a medida que avanza agosto y crece el sol, el blanco de las casas se hace más enceguecedor y adquieren las pieles un color más oscuro. (Bodas: 85)

On pourrait penser ici que même si le traducteur a pu proposer color, il est fort possible que dans une phase postérieure, un autre intermédiaire traductionnel, au nom des conventions descriptives, y ait vu une faute de frappe ou un lapsus (calor) color) et ait rétabli une perception plus «spontanée», plus "véridique» du rapport entre les choses, du comportement des objets dans un univers «objectif».

En ce qui concerne les exemples 9-11, l'intermédiaire traductionnel n'a pas cherché à effacer la synesthésie - dont l'interprétation est facilitée par l'information contextuelle - ; cependant il a réorganisé le cotexte immédiat où celle-ci s'inscrit de façon à créer des conditions favorables d'interaction en vue d'une interprétation stable ou bien de façon à restreindre son intensité et donc de sa force évocatoire:

(9) J'entrais dans le petit cabinet de repos [...] qui, même quand les fenêtres ouvertes laissaient entrer la chaleur, sinon les rayons du soleil qui atteignaient rarement jusquelà, dégageait inépuisablement cette odeur obscure et fraîche, à la fois forestière et ancien régime, qui fait rêver longuement les narines quand on pénètre dans certains pavillons de chasse abandonnés. (Du côté de chez Swann: 87)

Entraba en el cuarto de descanso [...] que, aunque las ventanas abiertas dejaran pasar el calor, ya que no los rayos solares, que no alcanzaban hasta allí, exhalaba sin cesar ese olor fresco y oscuro, a la vez forestal y antiguo régimen, que inspira largos sueños al olfato, cuando nos asalta al penetrar en un abandonado pabellón de caza.» (Por el carmino de Swann: 93)

La traduction de la synesthésie est ici lexicalement littérale; cependant, on constate une modification de l'ordre à l'intérieur du syntagme: l'inversion des adjectifs atténue quelque peu l'effet de la synesthésie (olor oscuro) en proposant en premier lieu l'association la plus traditionnelle et spontanée (olor fresco), éloignant ainsi la perception déroutante qui serait inscrite dans la proximité des termes olor et oscuro. Là encore il semblerait que l'intermédiaire traductionnel présuppose une attitude de lecture chez l'interlocuteur-cible qui ne serait qu'activité de déchiffrement en vue d'une compréhension sans qu'il y ait place pour le plaisir de ce déchiffrement ${ }^{11}$.

(10) Une matinée liquide se leva, éblouissante, sur la mer pure. Du ciel, frais comme un œil, lavé et relavé par les eaux, réduit par ces lessives successives à sa trame la plus fine et la plus claire descendait une lumière vibrante [...] (L'été: 160) 
Entonces amaneció una mañana líquida, deslumbrante, sobre el mar puro. Del cielo, fresco como un ojo lavado y vuelto a lavar por las aguas, reducido por aquellas sucesivas lejías a su trama más fina y más clara, descendía una luz vibrante $[\ldots]$. (El verano: 43 )

La double synesthésie (d'une part ciel et frais, et d'autre part la comparaison établie entre frais - perception tactile ou olfactive - et oeil) est atténuée dans le texte-cible, non dans le choix lexical, mais par la ponctuation: la suppression de la virgule après ojo déplace ce qui dans le texte original était les épithètes de ciel (lavé et relavé), et les assimile à des épithètes de ojo (lavado y vuelto a lavar), proposant ainsi un rapport linguistique non plus simplement entre fresco et ojo, mais entre fresco et ojo lavado ( fresco como algo recién lavado»), rejetant ainsi la suggestion originale présente dans la structure comparative.

(11) Oyó desde su cama el llanto de Úrsula, los pasos y murmullos de la multitud que invadió la casa, los aullidos de las plañideras, y luego un hondo silencio oloroso a flores pisoteadas. (Cien años de soledad: 93)

Elle entendit depuis son lit les sanglots d'Ursula, les pas et les murmures de la foule qui envahit la maison, les hurlements des pleureuses, et bientôt un profond silence plein du parfum des fleurs piétinées. (Cent ans de solitude: 121)

La synesthésie (silencio oloroso) est présente conceptuellement dans l'énoncécible, mais de façon moins franche, puisque l'adjectivation insolite est supprimée au profit d'une formule descriptive plus courante (plein de...) qui superpose et segmente en discontinu les sensations sans les enchaîner, perdant ainsi leur imbrication continue et donc la force évocatoire de la formulation originale qui, dans la traduction, pourrait éventuellement passer par un adjectif (parfumé, odorant). La formulation-cible libère en quelque sorte la tension de l'activité lectrice en proposant un élément à forte probabilité contextuelle.

Les synesthésies lexicalisées qui possèdent des équivalents également lexicalisés dans la langue-cible sont également soumises à un redressement de compatibilité sémantique comme on peut le constater dans les exemples 12-14:

(12) Le bleu tire aux flambeaux sur un faux vert; s'il est foncé comme le cobalt et l'indigo, il devient noir; s'il est clair, il tourne au gris; s'il est sincère et doux comme la turquoise, il se ternit et se glace. (À Rebours: 72)

El azul adquiere una tonalidad verdosa ante los focos de la luz; si es un azul oscuro, como el cobalto y el indigo, se vuelve negro; si se trata de un azul claro, se va haciendo casi gris, si es sincero y claro como el turquesa, entonces se apaga y se enfría. ( $A$ contrapelo: 133 )

L'extrait propose dans le texte-source la synesthésie lexicalisée couleur douce, qui possède un équivalent en espagnol: color suave: en effet, dans les deux langues doux/ suave fonctionne comme transposant sensoriel général applicable aux perceptions tactiles, visuelles, auditives, olfactives ${ }^{12}$. Cette synesthésie entre ici dans la comparaison «doux comme la turquoise». Bien que le terme turquoise soit au féminin (et fasse donc référence à la pierre, et non pas directement à la couleur, car lorsqu'il désigne une couleur, il est masculin), il est néanmoins possible de sous-entendre la (couleur) turquoise. Or on constate que la traduction propose une référence explicite à la couleur, mais que cependant elle rejette l'adjectif lexicalisé rétablissant une perception visuelle plus compatible avec la couleur (claro como el turquesa) dans ce qui 
semble être un souci d'éviter une hésitation et d'inscrire plus clairement le rapport chromatique.

(13) La saphirine qui allume des feux bleuâtres de phosphore sur un fond de chocolat, brun sourd. (À rebours: 98 )

La safirina que enciende fuegos azulones de fósforo sobre un fondo oscuro de chocolate. (A contrapelo: 169)

(14) Ils se mêlaient, les uns les autres, croisaient leurs épées, leurs kriss, leurs fers de lances, dessinaient un faisceau d'armes vertes, au-desssus duquel flottaient, ainsi que des fanions barbares, des fleurs aux tons aveuglants et durs. ( $\AA$ rebours: 137)

Se mezclaban los unos con los otros, cruzando sus espadas, sus puñales retorcidos, las puntas de sus lanzas, formando un pabellón de armas verdes, sobre el que aparecían flotantes como bárbaros estandartes, flores de tonos deslumbrantes e intensos. $(A$ contrapelo: 221)

Dans l'exemple 13, le traducteur a proposé une neutralisation explicative (fondo oscuro) plutôt qu'une expression qui aurait mis en œuvre, d'une façon ou d'une autre, un équivalent lexicalisé (color apagado) faisant image. Redressement renforcé par ailleurs par la disparition de la référence à la couleur et conséquemment par le déplacement de oscuro qui qualifie fondo. Une démarche similaire a lieu dans l'exemple 14 où tons durs n'est pas traduit par son équivalent lexicalisé (tonos/colores duros) mais par un adjectif cohérent avec l'adjectif antérieur, contribuant ainsi à homogénéiser la qualification.

Bien que l'intermédiaire traductionnel soit censé connaître les faits d'écriture, les spécificités et les complexités du texte littéraire, les exemples précédents démontrent, contrairement à ce que l'on pourrait attendre, qu'il existe une sorte de cloisonnement entre l'écriture littéraire et la réécriture traductionnelle comme s'il s'agissait de phénomènes indépendants et non interdépendants. Bien que l'on puisse penser que les contraintes du bon goût et de la compréhension ne soient plus considérées comme telles par ces intermédiaires, on constate néanmoins qu'ils demeurent porteurs d'un souci de vraisemblance des discours traduits, non pas en l'occurrence sous l'effet de leur propre imaginaire, mais bien plutôt sous le poids d'un "art d'écrire» officiel imprégné de tradition littéraire qui fait que "le réalisme et le naturalisme [...] se trouvent en quelque sorte reconnus comme la manière la plus "naturelle" » de regarder la vie, et promus comme l'écriture répondant le mieux aux exigences de la communication» (Abastado 1981: 270).

La prétention à la vérité extralinguistique de ces intermédiaires traductionnels leur fait perdre de vue la mise en écriture de l'imagination créatrice et les conduit à présenter les faits «comme ils devraient être» et non pas à reproduire une situation verbale, banalisant ainsi la spécificité des textes originaux, privilégiant la fonction référentielle du langage au détriment de la fonction poétique, et privilégiant conséquemment du lisible immédiatement accessible. Cette conception instrumentale du langage n'est pas sans rapport avec la scolarisation et la formation à la traduction (lorsqu'elle existe) le plus souvent axée sur le bon usage littéraire et le bon usage stylistique qui fait que la description axée sur l'observation - exercice de base de l'apprentissage de la rédaction/écriture - s'impose lors de la lecture du texte littéraire. Sous l'emprise du code descriptif entériné de type «réaliste», la description ressemblante ${ }^{13} s^{\prime}$ impose à son tour lors de la réécriture traductionnelle, comme si le lecteur-cible ne pouvait pas s'approprier l'univers de référence suscité par le texte et 
que le monde de la réalité devait s'imposer au monde de l'imaginaire, et comme si la connaisance sensible était plus difficile à appréhender que la connaissance analytique.

L'inscription des habitudes langagières et des clichés descriptifs dans les fragments analysés ${ }^{14}$ réduit l'expansion de l'imaginaire, limite les possibilités de chaque lecture singulière en gommant les segments où prennent appui les marques des itinéraires de lecture ${ }^{15}$, les nœuds de la motivation poétique. Cette pratique traduisante qui désigne le licite, le discours sensé, vise ainsi l'efficacité de la lecture — une limpidité et une crédibilité des énoncés fictifs —, et la maîtrise d'une capacité de déchiffrement d'un texte conduisant à la production d'une "histoire interprétable». Peut-on en déduire que la lecture initiale d'un récit, axée sur la progression textuelle, prédomine dans la réécriture traductionnelle? Et dans ce cas, pourquoi les autres dimensions de l'acte de lecture n'ont-elles pas de poids spécifiques pour les intermédiaires traductionnels? Pourquoi l'activité traductionnelle est-elle (encore trop souvent) orientée à la configuration d'un protocole de lecture qui va guider le lecteur-cible dans sa saisie du texte et dans son adhésion à l'univers narratif?

\section{NOTES}

1. Phénomènes que les écrivains ont signalé à maintes occasions. Rappelons ici, entre autres, les commentaires sur l'audition colorée de Baudelaire: «les sons ont une couleur, les couleurs ont une musique» (Les paradis artificiels (1860), Paris, Librairie générale française, coll. «Livre de poche», 1972, p. 47.); Hugo : «l'oreille aussi a sa vue» (Notre-Dame-de-Paris (1831) Paris, Librairie générale française, coll. «Livre de poche», 1972, p. 172.); le poème «Voyelles» de Rimbaud, etc.

2. «La synesthésie affecte surtout les adjectifs et les verbes, plus aptes que les noms à exprimer les transpositions de perception ou de qualité. Elle apparaît généralement comme une figure dérivée, ses transferts de sensations se greffant sur la métaphore ou sur la comparaison» (Bonhomme 1998: $68)$.

3. La synesthésie est une figure peu étudiée et généralement assimilée à la métaphore (métaphore synesthésique).

4. Le Guern (1973: 48) avait déjà signalé que la synesthésie est un cas très particulier d'image en ce sens qu'elle présente un caractère extralinguistique qu'une analyse sémique ne parvient pas à cerner, puisqu'elle met en jeu des correspondances dans la perception des différents sens «indépendamment des facultés linguistiques et logiques ». Pour le syntagme tintement doré, que l'on pourrait dans un premier temps rapporter à une synesthésie, il existe un rapport linguistique indirect: en effet, doré se définit comme "ce qui a l'éclat de l'or». Et éclat est un terme polysémique (intensité d'une lumière / bruit violent et soudain) qui permet d'établir un rapport avec tintement.

5. Les trois sources considérées par la pragmatique comme conduisant à des conclusions obtenues par inférence déductive.

6. Activité traductionnelle qui ne se résume bien entendu pas à une reproduction ou à une duplication du même. Je ne reviendrai pas ici sur ce débat.

7. «[... en la sinestesia, la cualidad irrealmente concedida al objeto resulta de una pura invención del autor» (Bousoño 1952: 159).

8. Choix d'autant plus surprenant que l'adjectif mordiente est traduit, dans ce même texte, par cuisant lorsqu'il entre dans d'autres figures rhétoriques.

9. Or dans les ouvrages de ce corpus, cette manipulation n'a pas lieu (sauf rares exceptions) pour les énoncés métaphoriques qui eux maintiennent l'inscription des images, bien que, dans ce cadrage réaliste des énoncés littéraires opéré par les intermédiaires traductionnels, il serait plausible de penser que métaphores et synesthésies portent la même atteinte à la description réaliste.

10. "Desplazamiento de las relaciones del adjetivo y del sustantivo. El adjetivo no concuerda ni gramaticalmente ni por su significado literal (sino por uno metafórico) con el sustantivo que le está contiguo, sino con otro, u otros, presentes dentro de un contexto inmediato» (Beristáin 1995: 248).

11. Il est également plausible de penser que la stratégie traductionnelle de Pedro Salinas (poète, ne l'oublions pas) correspond à une préférence pour un certain rythme de la phrase. Il est impossible de cerner ici ce phénomène qui serait éminemment intéressant à analyser. 
12. Seule la perception gustative propose un autre terme en espagnol (dulce).

13. À titre tout à fait anecdotique, mais qui me semble bien illustrer les remarques antérieures, je signale le passage suivant de M. Yourcenar («Comment Wang-Fô fut sauvé», in Nouvelles orientales, Paris, Gallimard, «Bibliothèque de la Pléiade», 1982, p. 1140.) : «Une nuit dans une taverne, il eut WangFô pour compagnon de table. Le vieil homme avait bu pour se mettre en état de mieux peindre un ivrogne.» La traduction espagnole interprète ce mieux peindre dans le sens de la description resemblante: "Una noche en una taberna, tuvo por compañero de mesa a Wang-Fô. El anciano había bebido, para ponerse en un estado que le permitiera pintar con realismo a un borracho" (Novelas orientales, Madrid, Alfaguara, 1989, p. 16-17).

14. L'analyse de ce mouvement dynamique du monde perceptuel du réécrivant, lors de la traduction des termes de couleurs, fait actuellement l'objet d'une recherche conjointe entre chercheurs de l'Université de Cadix et de l'École supérieure de traduction de Tanger (Maroc), financée par l'Agence espagnole de coopération internationale depuis 1998.

15. À l'inverse, je n'ai retrouvé qu'un seul exemple d'introduction de synesthésie là où le texte original n'en était pas porteur: "Le boulevard Diderot et plus loin l'enfilade de la rue de Lyon n'étaient qu'un scintillement de phares, d'enseignes, de vitrines, de terrasses de cafés, de feux tricolores » ( $L a$ goutte d'or: p. 116)/ «El bulevar Diderot, y un poco más lejos la enfilada de la calle de Lyon no eran sino un tintinear de faros, letreros, escaparates, terrazas de cafés, semáforos tricolores » (La gota de oro: p. 138).

\section{RÉFÉRENCES}

Abastado, C. (1981) «La composition française et l'ordre du discours », in Dérives des signes, Nanterre, Publidix, 1988, 263-283.

Arnheim, R. (1976) «Couleurs. Irrationnel et rationnel», Change, 26/27, p. 33-41.

Beristáin, H. (1985) Diccionario de retórica y poética, México, Editorial Porrúa, 1995.

Bonнomme, M. (1998) Les figures clés du discours, Paris, Seuil.

Bousoño, C. (1952) Teoría de la expresión poética, 2 vol., Madrid, Gredos, 1976.

Dupriez, B. (1977) Gradus. Les procédés littéraires Paris, Union générale d'éditions, 1980.

Gage, J. (1993) Colour and Cultur. Practice and Meaning from Antiquity to Abstraction, London, Thames and Hudson.

Gervais, B. (1990) Récits et actions. Pour une théorie de la lecture, Longueuil (Québec), Éditions du Préambule.

Guillerm, L. (1984) «L'intertextualité démontée: le discours sur la traduction», Littérature, 55, p. $54-63$.

Le Guern, M. (1973) Sémantique de la métaphore et de la métonymie, Paris, Larousse.

Ly, N. (dir.) (1992) Littéralité 2. Actes du colloque des 18-19 Mai 1990 organisé par le G.R.I.A.L., Presses universitaires de Bordeaux.

Mayoral, J. A. (1994) Figuras retóricas, Madrid, Editorial Síntesis.

Moeschler, J. et A. Reboul, (1994) Dictionnaire encyclopédique de pragmatique, Paris, Seuil.

Moreau, F. (1982) L'image littéraire, Paris, Sedes.

Morier, H. (1961) Dictionnaire de poétique et de rhétorique, Paris, PUF, 1981.

Sáez Hermosilla, T. (1987) «Reflexiones para una traductología del texto literario », Cuadernos de Traducción e Interpretación, 8/9, p.191-201.

Sperber, D. et D. Wilson (1986) «Façons de parler», Cahiers de linguistique française, 7, p. 9-26.

Vicente Cruz, B. (1993) «En torno a la semántica y la pragmática de la metáfora: una revisión crítica », Pragmalingüística, 1, Publicaciones de la Universidad de Cádiz, 307-334.

— La traduction. Actes du XXIII e Congrès de la Société des Hispanistes Français, Caen, 13-15 Mars 1987. Centre de publications de l'Université de Caen, 1989.

\section{Corpus}

Camus, A. (1939) Noces, L'été, Paris, Gallimard/coll. "Folio», 1981. El verano/Bodas, Barcelona, Pocket/Edhasa, 1986. Trad: Alberto Luis Bixio.

García Márquez, G. (1967) Cien años de soledad, Barcelona, Arcos Vergara, 1981. Cent ans de solitude, Paris, Seuils/coll. «Points», 1968. 
Hugo, V. (1862) Les misérables, Paris, Garnier-Flammarion, 1967, 3. vol. Los miserables, Barcelona, Círculo de Lectores, 1967. Trad: H. G. Simon.

Huysmans, J. (1884) À rebours, Paris, Garnier-Flammarion, 1978. A contrapelo, Madrid, Cátedra, 1984. Trad: Juan Herrero.

Proust, M. (1913 Du côté de chez Swann, Paris, Gallimard, coll. «Livre de Poche», 1954. Por el camino de Swann, Madrid, Alianza Editorial, 1966.

Rodenbach, G.(1892) Bruges-la-morte, texte établi d'après l'édition de 1892, Bruxelles, Labor, 1986. Brujas, la muerta, Madrid, Editorial Fortanet, 1918. Trad: Andrés Guilmain.

Tournier, M.(1985) La goutte d'or, Paris, Gallimard, coll. «Folio». La gota de oro, Madrid, Alfaguara, 1988. Trad: Jacqueline y Rafael Conte. 